Research Paper

\title{
New Oral Anticoagulants vs Vitamin K Antagonists: Benefits for Health-Related Quality of Life in Patients with Atrial Fibrillation
}

\author{
Josep M. Alegret ${ }^{\boxplus}$, Xavier Viñolas², Miguel A. Arias³, Antoni Martínez-Rubio4, Pablo Rebollo5, Carles \\ Ràfols ${ }^{6}$, José L. Martínez-Sande ${ }^{7}$ \\ 1. Grup de Recerca Cardiovascular, Hospital Universitari de Sant Joan, IISPV, Universitat Rovira i Virgili, Reus, Spain. \\ 2. Hospital de la Sta. Creu i St. Pau, Barcelona, Spain. \\ 3. Hospital Virgen de la Salud, Toledo, Spain. \\ 4. Hospital Universitari Parc Taulí, Sabadell, Spain. \\ 5. LA-SER Outcomes, Oviedo, Spain. \\ 6. Medical Department, Bayer HealthCare, Barcelona, Spain \\ 7. Hospital Clínico, Santiago de Compostela, Spain.
}

$\triangle$ Corresponding author: Josep M Alegret, Grup de Recerca Cardiovascular, Hospital Universitari de Sant Joan, Departament de Medicina i Cirurgia, Universitat Rovira i Virgili. c/ Dr Josep Laporte, n¹, 43204 Reus. Email: josepmaria.alegret@urv.net.

(c) Ivyspring International Publisher. This is an open-access article distributed under the terms of the Creative Commons License (http://creativecommons.org/ licenses/by-nc-nd/3.0/). Reproduction is permitted for personal, noncommercial use, provided that the article is in whole, unmodified, and properly cited.

Received: 2014.02.24; Accepted: 2014.04.01; Published: 2014.05.01

\begin{abstract}
New oral anticoagulants (NOAC) have demonstrated their efficacy as an alternative to vitamin $\mathrm{K}$ antagonists (VKA) in the prophylaxis of cardioembolic events in patients with atrial fibrillation (AF). However, evidence on the benefits of NOAC in health-related quality of life (HRQoL) is lacking.We evaluated changes in $\mathrm{HRQ}$ oL related to oral anticoagulation therapy employing a specific questionnaire in a cohort of 416 patients with AF undergoing electrical cardioversion. In terms of HRQoL, we observed a progressive adaptation to treatment with VKA; satisfaction with NOAC remained constant. Older age, higher left ventricular ejection fraction and NOAC were associated with better HRQoL.
\end{abstract}

Key words: vitamin K antagonists; dabigatran; health-related quality of life; new oral anticoagulants; ribaroxaban.

\section{Introduction}

Treatment with vitamin $\mathrm{K}$ antagonists (VKA) is complex and requires frequent analytical work-ups and visits to monitor the international normalized ratio (INR). This treatment can affect patients' assessment of health-related quality of life (HRQoL) in that it requires changes in lifestyle and not provide relief from symptoms [1]. Results from clinical trials have demonstrated the usefulness of new oral anticoagulants (NOAC) (dabigatran, rivaroxaban, and apixaban) as an alternative to conventional treatment with VKA in the prophylaxis of cardioembolic events in patients with AF [2-4]. NOAC have shown also its benefits in specific settings as electrical cardioversion [5] and catheter ablation of AF [6]. One of the benefits of therapy with NOAC is that it obviates the periodi- cal monitoring required for VKA. Consequently, assessment of HRQoL in patients with AF treated with NOAC could improve. However, evidence of the superiority of NOAC over VKA in HRQoL is lacking.

In the present study, we compared the impact of NOAC on HRQoL with that of conventional treatment based on VKA in a group of patients with AF undergoing electrical cardioversion who had recently initiated anticoagulant treatment.

\section{Methods}

\section{Patients}

HRQoL was evaluated at cardioversion (baseline) and 6 months later in 416 patients included in the CARDIOVERSE study. The CARDIOVERSE study 
was designed to monitor the clinical practice of elective electrical cardioversion in Spain by prospectively recording all patients with persistent $\mathrm{AF}$ who underwent the procedure between $1^{\text {st }} \mathrm{Feb}$ and $30^{\text {th }}$ June 2012 in 67 Spanish hospitals. Patients were recruited consecutively. The inclusion criteria were age $>18$ years, duration of $\mathrm{AF}>7$ days, and absence of AF precipitating conditions (e.g., hyperthyroidism, fever, and pericarditis). No instructions for the use of anticoagulant treatment had been recommended. For the current analysis, we selected those patients who had initiated anticoagulant therapy in the 4 months before electrical cardioversion $(\mathrm{N}=528)$ and who completed a questionnaire on HRQoL ( $\mathrm{N}=416)$. Two patients with disabling embolic or hemorrhagic events during follow-up were excluded. We divided patients into 2 groups, depending on whether they had received NOAC $(n=65)$ or VKA $(n=351)$. At the beginning of the study, the only NOAC approved in Spain for the prophylaxis of embolisms in AF was dabigatran; rivaroxaban was approved at the end of the recruitment period. Fifty-nine patients were treated with dabigatran and 6 patients were treated with rivaroxaban. Apixaban was not approved for this indication in Spain during the present study.

\section{Questionnaire and assessment of HRQoL}

We analyzed the scores of a specific questionnaire designed to assess HRQoL in patients treated with oral anticoagulants. The questionnaire used was a validated Spanish adaptation [7] of the Sawicki questionnaire [8], an original questionnaire that has been used in several studies [9-11]. The questionnaire includes 32 items grouped in 5 dimensions. Patients estimated the impact of each item on their self-perceived treatment-related quality of life on a scale of 1 (total disagreement) to 6 (total agreement). The 5 dimensions are general treatment satisfaction, self-efficacy, strained social network, daily hassles, and distress. The self-efficacy dimension was not analyzed in the present study owing to the characteristics of NOAC. A higher score in the dimensions indicates greater impact and, therefore, worse situation of the individual patient. Finally, we obtained a global score by adding up the score of each dimension. A lower score indicates higher HRQoL and a higher score indicates lower HRQoL. The questionnaire was self-completed.

Continuous variables are expressed as mean $\pm S D$, and comparisons between groups were made using the $t$ test (for independent values when comparing both groups at baseline and at 6 months and for paired values when comparing the same group at both time points). Categorical variables are expressed as percentages and were analyzed using the chi-square test. Stepwise multiple linear regression models were developed to find independent factors related to the global score of HRQoL. Gender, age, left ventricular ejection fraction, diabetes mellitus, NYHA, congestive heart failure, CHADS2, CHA2DS2VASc, HAS-BLED and NOAC were included in the analysis. The internal reliability of the questionnaire was assessed using the Cronbach alpha coefficient. Differences were considered statistically significant if $p<0.05$. The statistical analysis was performed using SPSS Inc. Released 2009. PASW Statistics for Windows, Version 18.0. Chicago: SPSS Inc.

The study was approved by the Institutional Review Boards (Clinical Ethics Committee) of each participating hospital. Written informed consent was obtained from all the patients who participated in this study.

\section{Results}

The baseline characteristics of the 416 patients analyzed are presented in Table 1 . No significant differences were detected between patients treated with VKA and patients treated with NOAC in terms of duration of therapy, maintenance of sinus rhythm, or other characteristics potentially related to HRQoL.

Table 2 shows the questionnaire scores. At baseline, the general treatment satisfaction score was significantly lower and the daily hassles score tended to be significantly lower in the NOAC group (better HRQoL). In addition, the global score tended to be lower in the NOAC group (also indicating a better HRQoL) (10.3 \pm 3.5 COA vs 9.6 \pm 3.7 NOAC; $p=0.17)$. Six months after cardioversion, 252 patients continued to receive oral anticoagulant therapy and completed the questionnaire (215 in the VKA group and 37 in the NOAC group). Scores for general treatment satisfaction, daily hassles, distress, and strained social network improved in the VKA group with respect to baseline and did not show significant changes in the NOAC group (Table 3 ). When we compared thescores for both groups at 6 months, we did not observe significant differences in any dimension (Table 2). The global score was similar in both groups at 6 months (9.5 \pm 3.5 VKA vs $9.4 \pm 3.0$ NOAC; $p=0.88)$.

The internal reliability was acceptable as indicated by the Cronbach $\alpha$ values. A ceiling effect (more than $15 \%$ of maximum value for a dimension) was only observed in strained social network at baseline $(19 \%)$.

We applied multiple linear regressions to investigate those variables independently associated with the global score at baseline. Older age $(\beta=-0.05 \times$ year; $\mathrm{p}=0.009)$, higher left ventricular ejection fraction $(\beta=$ $-0.05 \times \% ; p=0.002)$, and NOAC $(\beta=-0.56 ; p=0.03)$ were associated with a lower global score (better HRQoL). 
Table I. Characteristics of patients.

\begin{tabular}{|c|c|c|c|c|}
\hline & & VKA $n=351$ & NOAC $n=65$ & $p$ \\
\hline Age, years & & $63.1 \pm 10.1$ & $61.0 \pm 9.5$ & 0.13 \\
\hline Male gender, $\mathrm{n}(\%)$ & & $285(81)$ & $58(89.2)$ & 0.09 \\
\hline BMI, kg/m² & & $29.0 \pm 4.3$ & $29.3 \pm 4.2$ & 0.70 \\
\hline $\mathrm{LA}, \mathrm{mm}$ & & $44 \pm 6$ & $43 \pm 5$ & 0.61 \\
\hline LVEF - quantitative & & $58 \pm 11$ & $60 \pm 9$ & 0.12 \\
\hline \multirow[t]{4}{*}{ LVEF - qualitative, n (\%) } & Normal $(\geq 50 \%)$ & $260(80)$ & $54(89)$ & 0.30 \\
\hline & Slightly depressed (49-41\%) & $32(9)$ & $5(8)$ & \\
\hline & Moderately (40-31\%) & $22(7)$ & $1(2)$ & \\
\hline & Severely $(\leq 30 \%)$ & $13(4)$ & $1(2)$ & \\
\hline Hypertension, n (\%) & & $206(57)$ & $39(60)$ & 0.79 \\
\hline Diabetes mellitus, n (\%) & & $58(17)$ & $16(25)$ & 0.11 \\
\hline Pulmonary disease, n (\%) & & $38(11)$ & $6(9)$ & 0.72 \\
\hline Congestive heart failure, n (\%) & & $42(12)$ & $12(19)$ & 0.15 \\
\hline Previous electrical CV, n (\%) & & $45(13)$ & $5(8)$ & 0.25 \\
\hline Antiplatelet therapy, n (\%) & & $45(13)$ & $7(11)$ & 0.66 \\
\hline \multirow[t]{3}{*}{$\mathrm{CHADS}_{2}, \mathrm{n}(\%)$} & Low risk $($ score $=0$ ) & $113(32)$ & $18(28)$ & 0.70 \\
\hline & Medium risk (score $=1$ ) & $146(42)$ & $26(40)$ & \\
\hline & High risk (score $\geq 2$ ) & $92(26)$ & $21(32)$ & \\
\hline \multirow[t]{3}{*}{$\mathrm{CHA}_{2} \mathrm{DS}_{2} \mathrm{VASc}, \mathrm{n}(\%)$} & Low risk $($ score $=0$ ) & $68(19)$ & $13(20)$ & 0.98 \\
\hline & Low-medium risk $($ score $=1$ ) & $96(27)$ & $17(26)$ & \\
\hline & Medium-high risk (score $\geq 2$ ) & $187(53)$ & $35(54)$ & \\
\hline \multirow[t]{2}{*}{ HAS-BLED, n (\%) } & Low-medium risk $($ score $<3)$ & $335(95)$ & $60(92)$ & 0.66 \\
\hline & High risk (score $\geq 3$ ) & $16(5)$ & $5(8)$ & \\
\hline \multirow[t]{5}{*}{ Duration of $\mathrm{AF}, \mathrm{n}(\%)$} & 8 days -1 month & $15(4)$ & $4(6)$ & 0.66 \\
\hline & 1 - 3 months & $131(37)$ & $22(34)$ & \\
\hline & 3 - 12 months & $122(35)$ & $19(29)$ & \\
\hline & $>12$ months & $25(7)$ & $7(11)$ & \\
\hline & Unknown & $58(17)$ & $13(20)$ & \\
\hline \multirow{5}{*}{$\begin{array}{l}\text { Symptoms related to } \mathrm{AF}, \mathrm{n}(\%) \\
\text { (EHRA scale) }\end{array}$} & I & $145(41)$ & $33(51)$ & 0.18 \\
\hline & II & $183(52)$ & $26(40)$ & \\
\hline & III & $17(5)$ & $5(8)$ & \\
\hline & IV & $1(0.5)$ & $1(1)$ & \\
\hline & Unknown & $5(1.5)$ & $0(0)$ & \\
\hline
\end{tabular}

Values represent mean $\pm \mathrm{SD}$ or $\mathrm{n}(\%)$. AF, atrial fibrillation; BMI, body mass index; VKA, vitamin K antagonists; CV, cardioversion; EHRA, European Heart Rhythm Association; LA, left atrium; LVEF, left ventricle ejection fraction; NOAC, new oral anticoagulant.

Table 2. Comparison of questionnaire scores at baseline and at 6 months in relation to the type of oral anticoagulant treatment.

\begin{tabular}{|c|c|c|c|c|c|c|c|c|c|c|}
\hline & \multicolumn{5}{|c|}{ Baseline } & \multicolumn{5}{|c|}{ Follow-up (6 months) } \\
\hline & Cronbach a & VKA & NOAC & Difference & $p$ & Cronbach a & VKA & NOAC & Difference & $p$ \\
\hline General treatment satisfaction & 0.75 & $2.5 \pm 1.0$ & $2.2 \pm 1.1$ & -0.3 & $<0.01$ & 0.75 & $2.3 \pm 1.0$ & $2.2 \pm 1.0$ & -0.1 & 0.80 \\
\hline Distress & 0.84 & $3.3 \pm 1.1$ & $3.2 \pm 1.0$ & -0.1 & 0.46 & 0.84 & $3.1 \pm 1.0$ & $3.1 \pm 0.9$ & 0 & 0.86 \\
\hline Daily hassles & 0.81 & $2.3 \pm 1.0$ & $2.2 \pm 1.1$ & -0.1 & 0.13 & 0.78 & $2.1 \pm 1.0$ & $2.0 \pm 0.9$ & -0.1 & 0.91 \\
\hline Strained social network & 0.72 & $2.0 \pm 1.0$ & $1.9 \pm 1.0$ & -0.1 & 0.23 & 0.68 & $1.9 \pm 0.9$ & $2.0 \pm 0.8$ & 0.1 & 0.29 \\
\hline
\end{tabular}

VKA, vitamin K antagonists; NOAC, new oral anticoagulant.

Table 3. Changes in the questionnaire scores between baseline and at 6 months depending on the type of oral anticoagulant treatment.

\begin{tabular}{|c|c|c|c|c|c|c|c|c|}
\hline & \multicolumn{4}{|c|}{ VKA } & \multicolumn{4}{|c|}{ NOAC } \\
\hline & Baseline & 6 months & Difference & $p$ & Baseline & 6 months & Difference & $p$ \\
\hline General treatment satisfaction & $2.5 \pm 1.0$ & $2.3 \pm 1.0$ & -0.2 & $<0.01$ & $2.4 \pm 1.2$ & $2.2 \pm 1.0$ & -0.2 & 0.48 \\
\hline Distress & $3.3 \pm 1.1$ & $3.1 \pm 1.0$ & -0.2 & $<0.001$ & $3.3 \pm 1.1$ & $3.1 \pm 0.9$ & -0.2 & 0.24 \\
\hline Daily hassles & $2.3 \pm 1.1$ & $2.1 \pm 1.0$ & -0.2 & $<0.005$ & $2.2 \pm 1.1$ & $2.0 \pm 0.9$ & -0.2 & 0.45 \\
\hline Strained social network & $2.1 \pm 1.0$ & $1.9 \pm 0.9$ & -0.2 & $<0.05$ & $1.9 \pm 2.0$ & $2.0 \pm 0.8$ & 0.1 & 0.43 \\
\hline
\end{tabular}

VKA, vitamin K antagonists; NOAC, new oral anticoagulant.

\section{Discussion}

At initiation of anticoagulant therapy in patients with $\mathrm{AF}$, we observed a worse HRQoL in some dimensions in patients treated with conventional VKA than in patients treated with NOAC. However, these differences disappeared 6 months later. We identified age, left ventricular ejection fraction, and treatment with NOAC as factors independently associated with better HRQoL.

The greatest differences in HRQoL were observed in the general treatment satisfaction dimen- 
sion. The lower perception of HRQoL during the first months of VKA treatment with respect to NOAC may be explained by the higher number of visits required at the beginning of therapy and the frequent difficulties in achieving adequate INR levels [12-14]. The subsequent lower requirement for visits and more stable levels of INR could justify the improvement in the assessment of HRQoL and the lack of differences between the NOAC and VKA groups at 6 months.

Some variables were associated with perception of HRQoL. A worse left ventricular function was associated with worse HRQoL, probably because anticoagulation therapy created more problems for patients with an already reduced HRQoL. Aging was directly related to the HRQoL level. Younger patients showed lower levels of HRQoL related to anticoagulation treatment than older patients. Independence in daily social and working activities and avoidance of medical visits seem to be of particular concern to younger patients receiving anticoagulant therapy. This is especially true for employed persons. Our results show that NOAC improves these restrictions with respect to conventional treatment with VKA. Although we included patients undergoing electrical cardioversion, we believe that our results could be applied in other groups, such as patients undergoing catheter ablation of $\mathrm{AF}$ and patients for whom a rate control strategy is chosen.

Our study is subject to a series of limitations. First, the questionnaire used was originally designed for assessment of HRQoL associated with VKA treatment. Consequently, other aspects related to HRQoL in AF may be not detected. HRQoL affected by damage arising from disabling embolic or hemorrhagic events was not assessed in this questionnaire. In fact, 2 patients who had experienced these types of events were excluded from the analysis. In a sub-group of patients participating in the RE-LY trial, Monzet al. used a general non-specific disease questionnaire, the EQ-5D, and a visual analog scale to compare the impact of dabigatran on HRQoL with that of warfarin [15]. They found no significant differences in HRQoL between patients treated with dabigatran and patients treated with warfarin. In contrast, our objectives were different, the focus of our study being the association between HRQoL and anticoagulant therapy itself. Our findings suggest that our approach is more realistic for detection of differences in HRQoL associated with NOAC. Second, there is a clear imbalance in the number of patients included in the treatment groups. This distribution is coherent with the current use of the anticoagulation treatment in Europe [16]. The decrease in the number receiving NOAC at 6 months limits the possibility of establishing significant differences. Third, our study was not randomized. However, it included consecutive patients included in different hospitals and it reflects real-life assessment of HRQoL. Finally, patients treated with NOAC received mainly dabigratan. Therefore, these conclusions should be applied strictly to this drug. The potential advantages of rivaroxaban over dabigatran include once-daily dosing (compared with dabigatran, which is administered twice daily) and lower non-bleeding upper gastrointestinal side effects (recorded in $16.9 \%$ of individuals receiving dabigatran in the RE-LY trial [RR vs warfarin $=1.81$; $\mathrm{p}$ $<0.001]$ [17]. These advantages could have a favorable effect on HRQoL, although the small number of patients included prevents analysis. Our study did not analyze other aspects related to patient preferences, such as drug price.

In conclusion, in terms of HRQoL, we observed a progressive adaptation to VKA. Satisfaction with NOAC remained unchanged. Older age, higher left ventricular ejection fraction and NOAC were associated with better HRQoL.

\section{Supplementary Material}

List of centers and investigators participating in the study. http://www.medsci.org/v11p0680s1.pdf

\section{Acknowledgment}

Authors would acknowledge Almudena Gonzalez Amo and Raúl Beltrán-Debón for their invaluable administrative assistance.

\section{Competing Interests}

This study was funded by Bayer Health Care, Spain.

JMA has not added conflict of interest to declare. $\mathrm{XV}$ has exerted as consultant for Bayer. MAA has participated in conferences for Bayer. AM-R has received research grants, and has participated in advisory boards and conferences for Bayer, Boehringer Ingelheim and Pfizer. PR has not added conflict of interest to declare. CR is employee of Bayer.

\section{References}

1. Wokhlu A, Monahan KH, Hodge DO, Asirvatham SJ, Friedman PA, Munger TM et al. Long-term quality of life after ablation of atrial fibrillation the impact of recurrence, symptom relief, and placebo effect. J Am CollCardiol. 2010; 55: 2308-2316

2. Connolly SJ, Ezekowitz D, Yusuf S,Eikelboom J, Oldgren J, Parekh A et al. Dabigatran versus warfarin in patients with atrial fibrillation. $N$ Engl J Med 2009; 361: 1139-1151.

3. Patel MR, Mahaffey KW, Garg J, Pan G, Singer DE, Hacke Wet al. ROCKET AF Investigators. Rivaroxaban versus warfarin in nonvalvular atrial fibrillation. $N$ Engl J Med 2011; 365: 883-891.

4. Granger CB, Alexander JH, McMurray JJ, Lopes RD, Hylek EM, Hanna M et al. ARISTOTLE Committees and Investigators. Apixaban versus warfarin in patients with atrial fibrillation. N Engl J Med 2011; 365: 981-992.

5. Piccini JP, Stevens SR, Lokhnygina Y, Patel MR, Halperin JL, Singer DE et al. Outcomes after cardioversion and atrial fibrillation ablation in patients treated with rivaroxaban and warfarin in the ROCKET AF trial. J Am CollCardiol 2013;61:1998-2006 
6. Providência R, Albenque JP, Combes S, Bouzeman A, Casteigt B, Combes N et al. Safety and efficacy of dabigatran versus warfarin in patients undergoing catheter ablation of atrial fibrillation: a systematic review and meta-analysis. Heart 2014;100:324-35.

7. Sánchez González R, Yanes Baonza M, Cabrera Majada A, Ferrer García-Borrás JM,Alvarez Nido R, Barrera Linares E. Adaptación transcultural de un cuestionario para medir la calidad de vida de los pacientes con anticoagulación oral. Aten Primaria 2004; 34: 353-359.

8. Sawicki PT. A structured teaching and self-management program for patients receiving oral anticoagulation: a randomized controlled trial. Working group for the Study of Patient Self-Management of Oral Anticoagulation. JAMA 1999; 281: $145-50$.

9. Gadisseur AP, Kaptein AA, Breukink-Engbers WG,de Mol BJ, Prins MH, Hutten BA et al. Patient self-management of oral anticoagulant care vs. management by specialized anticoagulation clinics: positive effects on quality of life. J ThrombHaemost 2004; 2: 584-591.

10. Cromheecke ME, Levi M, Colly LP, de Mol BJ, Prins MH, Hutten BA et al. Oral anticoagulation self-management and management by a specialist anticoagulation clinic: a randomised cross-over comparison. Lancet 2000; 356: 97-102.

11. Siebenhofer A, Hemkens LG, Rakovac I, Spat S, Didjurgeit U. Self-management of oral anticoagulation in elderly patients - effects on treatment-related quality of life. Thromb Res 2012; 130: e60-66.

12. Connolly S, Pogue J, Hart R, Pfeffer M, Hohnloser S, Chrolavicius S et al; the ACTIVE Writing Group on behalf of the ACTIVE Investigators. Clopidogrel plus aspirin versus oral anticoagulation for atrial fibrillation in the Atrial fibrillation Clopidogrel Trial with Irbesartan for prevention of Vascular Events (ACTIVE W): a randomised controlled trial. Lancet 2006;367:1903-1912.

13. Mant J, Hobbs FDR, Fletcher K, Roalfe A, Fitzmaurice D, Lip GYH et al; on behalf of the BAFTA investigators and the Midland Research Practices Network (MidReC). Warfarin versus aspirin for stroke prevention in an elderly community population with atrial fibrillation (the Birmingham Atrial Fibrillation Treatment of the Aged Study, BAFTA): a randomized controlled trial. Lancet 2007;370:493-503.

14. Hylek EM, Evans-Molina C, Shea C, Henault LE, Regan S. Major hemorrhage and tolerability of warfarin in the first year of therapy among elderly patients with atrial fibrillation. Circulation 2007;115:2689-2696.

15. Monz BU, Connolly SJ, Korhonen M, Noack H, Pooley J. Assessing the impact of dabigatran and warfarin on health-related quality of life: Results from an RE-LY sub-study. Int J Cardiol 2013;168:2540-7.

16. Lip GY, Laroche C, Dan GA, Santini M, Kalarus Z, Rasmussen LH et al. A prospective survey in European Society of Cardiology member countries of atrial fibrillation management: baseline results of EURObservational Research Programme Atrial Fibrillation (EORP-AF) Pilot General Registry. Europace 2014 [Epub ahead of print].

17. Bytzer P, Connolly SJ, Yang S, et al. Analysis of upper gastrointestinal adverse events among patients given dabigatran in the RE-LY trial. ClinGastroenterolHepatol 2013;11:246-52. 